\title{
ON THE STRUCTURE OF CONNECTED AND CONNECTED IM KLEINEN POINT SETS*
}

\author{
BY \\ G. T. WHYBURN
}

\section{INTRODUCTION; NOTATION; SOME KNOWN RESULTS}

In this paper it is shown that all connected and connected im kleinen point sets lying in a separable metric space admit of decomposition into elements, called nodular elements, which are highly analogous to the cyclic elements of a continuous curve $\dagger$ and that the structure of these sets relative to their nodular elements is very similar to the structure of continuous curves relative to their cyclic elements. Indeed, it is true that for the special case of a continuous curve $M$, the nodular elements of $M$ are identically the same as the cyclic elements of $M$.

The usual terminology and notation of point set theory will be employed. To facilitate the reading of the paper, however, some of the less familiar terms and notations used are here defined and explained. The point $P$ of a connected point set $M$ is a non-cut point or a cut point of $M$ according as $M-P$ is or is not connected. The subset $\dot{X}$ of a connected set $M$ separates two subsets $A$ and $B$ of $M$ in $M$ provided that $M-X$ is the sum of two mutually separated sets $M_{a}(X)$ and $M_{b}(X)$ containing $A$ and $B$ respectively, and this fact is indicated by means of the equation $M-X=M_{a}(X)+M_{b}(X)$. The subset $K$ of a point set $M$ is said to be closed relative to $M$, or closed in $M$, provided that no point of $M-K$ is a limit point of $K$. The symbol $\supset$ means "contains," and c means "is contained in"; $\delta(H)$ denotes the diameter of the point set $H$, and $\rho(X, Y)$ denotes the lower bound of the aggregate of numbers $[\rho(x, y)]$, where $x$ and $y$ are points of $X$ and $Y$ respectively and $\rho(x, y)$ is the distance from $x$ to $y$.

Since this paper is concerned very largely with connected and connected im kleinen sets, it is to be understood, unless definitely stated to the contrary, that when we speak of a "set $M$ " it is presupposed that $M$ is a connected and connected im kleinen point set. The following results, some of which will be used later, either are already known or follow, as indicated below, quite readily from known properties of connected im kleinen point sets.

* Presented to the Society, December 27, 1928; received by the editors in October, 1929.

† See my paper Concerning the structure of a continuous curve, American Journal of Mathematics, vol. 50 (1928), pp. 167-194. 
(1.1) If $A$ and $B$ are two points of $a$ set $M$ and $K$ is the set of all those points of $M$ which separate $A$ and $B$ in $M$, then $K+A+B$ is a closed and compact point set.

(1.2) Using the same notation as in (1.1), with $M$ any connected point set, the points of $K$ are linearly ordered from $A$ to $B$ in $M$; and if $H$ is any closed and compact subset of $K$, there exists a first point of $H$ in the order from $A$ to $B$.

(1.3) The set $G$ of all the cut points of. a set $M$ is an $F_{\sigma}$ set, i.e., the sum of a countable number of closed sets.

(1.4) If the two points $A$ and $B$ of a set $M$ are not separated in $M$ by $a$ subset $X$ of $M$ which is closed in $M$ and contains neither $A$ nor $B$, then $A$ and $B$ lie together in a connected subset of $M-X$.

Result (1.1) has been proved by the author for a less general space elsewhere.* The proof there given for the closure of the set $K+A+B$ holds equally well in the space considered in the present paper. That $K+A+B$ is compact is proved in general as follows: Suppose, on the contrary, that $K+$ $A+B$ contains an infinite subset $D$ having no limit point. Now for each point $X$ of $K, M-X=M_{a}(X)+M_{b}(X)$, where the sets $M_{a}(X)$ and $M_{b}(X)$ are mutually separated and contain $A$ and $B$.respectively. As shown in my paper just cited, the set $D$ contains a sequence $X_{1}, X_{2}, X_{3}, \cdots$ of distinct points of $K$ such that either for each $i, X_{i} \subset M_{b}\left(X_{i-1}\right)$, or for each $i, X_{i} \subset$ $M_{a}\left(X_{i-1}\right)$. The two cases are alike, so we shall suppose the former. Now the set of points $H=\sum_{1}^{\infty} M_{a}\left(X_{i}\right)$ is closed in $M$, because the set $\sum_{1}^{\infty} X_{i}$ is closed and is a subset of $H$, and $M$, by hypothesis, is connected im kleinen. But $H$ is also open in $M$, since the sets $M_{a}\left(X_{i}\right)$ are open in $M$. This is impossible, because $H \neq M$ and $M$ is connected.

The first part of result (1.2) has also been proved by the author elsewhere. $\dagger$ The second part of this result follows readily from the first part of (1.2) and the fact that $H$ is closed, compact, and separable. Result (1.3) follows from (1.1) and the fact that $M$ is separable by an argument similar to, but simpler than, that used by Zarankiewicz $\ddagger$ to prove the same theorem for the special case where $M$ is a continuous curve. I note here the fact that

* See Concerning connected and regular point sets, Bulletin of the American Mathematical Society, vol. 33 (1927), p. 685. For a very simple proof of the same theorem, the closure part of which also is valid in the space considered in this paper, see R. L. Wilder, On connected and regular point sets, ibid., vol. 34 (1928), pp. 649-655.

† See Concerning collections of cuttings of connected point sets, Bulletin of the American Mathematical Society, vol. 35 (1929), pp. 90, 91.

$\ddagger$ See Sur les points de division dans les ensembles connexes, Fundamenta Mathematicae, vol. 9 (1927). 
it is also true that the set $K$ of all the im kleinen cut points of a set $M$ (i.e., all points $P$ of $M$ such that $P$ is a cut point of some connected open subset of $M$ ) is an $F_{\sigma}$ set. Result (1.4) is due to R. L. Wilder.*

\section{Preliminary lemmas}

(2.1) If $K$ is any closed (in $M$ ) subset of a connected and connected im kleinen point set $M$ having the property that, for each component $N$ of $M-K$, $\bar{N} \cdot K$ consists of a single point, then for every connected subset $H$ of $M, H \cdot K$ is either vacuous or connected.

Suppose, on the contrary, that, for some connected subset $H$ of $M$, $H \cdot K=H_{1}+H_{2}$, where $H_{1}$ and $H_{2}$ are mutually separated point sets. Let $G$ be the collection of all those components of $M-K$ each of which contains at least one point of $H$. For each element $g$ of $G$, it is easily seen that the point $P_{o}=\bar{g} \cdot K$ is a point of $H \cdot K$. Let $G_{1}$ be the collection of elements $g_{1}$ of $G$ such that $P_{g_{1}} \subset H_{1}$ and $G_{2}$ the collection of elements $g_{2}$ of $G$ such that $P_{g_{2}} \subset H_{2}$. Let

$$
M_{1}=H_{1}+H \cdot \sum_{g_{1 i} \text { in } G_{1}} g_{1 i} \text { and } M_{2}=H_{2}+H \cdot \sum_{0_{2 i} \text { in } G_{2}} g_{2 i} .
$$

Then clearly $M_{1}$ and $M_{2}$ are mutually exclusive and $M_{1}+M_{2}=H$. They must also be mutually separated. For suppose one of them contains a limit point of the other, say $M_{1}$ contains a limit point $P$ of $M_{2}$. Now since $M$ is connected im kleinen and $K$ is closed in $M$, therefore each component of $M-K$ is an open subset of $M$; and thus it follows that $P$ does not belong to $H \cdot \sum g_{1 i}$. Therefore $P \subset H_{1}$. Since $H_{1}$ and $H_{2}$ are mutually separated, there exists a neighborhood $R$ of $P$ such that $\bar{R} \cdot H_{2}=0$. But now since $M$ is connected im kleinen and $P$ is a limit point of $M_{2}$, there exists a point $Q$ of $M_{2}$ belonging to a component $g_{2}$ of $M-K$, which lies together with $P$ in a connected subset $I$ of $M \cdot R$. It readily follows that $R \cdot K$ contains a point $X$ which is a limit point of $g_{2}$. But $P_{g_{2}}$ does not belong to $R$, and by hypothesis $P_{o_{2}}$ is the only point in $\overline{\mathrm{g}}_{2} \cdot K$. Thus the supposition that $H \cdot K$ is not connected leads to a contradiction.

(2.2) Under the conditions of (2.1), $K$ is connected and connected im kleinon.

By letting $M=H$ in (2.1), we get that $M \cdot K=K$ is connected. If $P$ is any point of $K$ and $\epsilon>0$, then since $M$ is connected im kleinen, a $\delta_{\epsilon}>0$ exists such that each point $Q$ of $K$ with $\rho(Q, P)<\delta_{\epsilon}$ lies together with $P$ in a con-

* See $A$ characterization of continuous curves by a property of their open subsets, Fundamenta Mathematicae, vol. 11 (1928), pp. 127-131. 
nected subset $I$ of $M$ with $\delta(I)<\epsilon$. Then since, by (2.1), $I \cdot K$ is connected and contains $P+Q$, it follows that $K$ is connected im kleinen at each of its points $P$.

For convenience of reference we state here also the following easily established lemma which was stated to me in less general form by R. L. Moore.

(2.3) If $H$ is any subset of a connected point set $M$, then the set $K$ of all those points of $M$ which are not separated in $M$ from any point of $H$ by any single point of $M$ is closed in $M$.

\section{Nodular SUbSETS}

Definitions. A connected subset $C$ of a connected point set $M$ will be called "nodular" provided that no point of $C$ is a cut point of $C$. A nodular subset $C$ of $M$ is called a maximal nodular subset of $M$ provided that $C$ is saturated with respect to the property of being a nodular subset of $M$.

(3.1) Every maximal nodular subset $C$ of a connected. and connected im kleinen set $M$ is closed (in $M$ ) and connected.

By definition, $C$ is connected. Suppose $P$ is any limit point of $C$ which belongs to $M$. Then $P$ must belong to $C$. For since $C$ has no cut point, obviously $C+P$ has no cut point. Then $C+P$ is a nodular subset of $M$ containing $C$ as a proper subset, contrary to the fact that $C$ is a maximal nodular subset of $M$.

(3.2) A nodular subset $C$ of a connected and connected im kleinen set $M$ is maximal if $C$ is closed (in $M$ ) and no component of $M-C$ has more than one limit point in $C$.

Suppose, on the contrary, that $C$ is a proper subset of some nodular subset $C_{0}$ of $M$. Let $N$ be a component of $M-C$ containing a point $P$ of $C_{0}-C$. Since $C$ is closed and $M$ is connected im kleinen, $N$ must have at least one limit point $Q$ in $C$; and by hypothesis $Q$ is the only limit point of $N$ in $C$. Then, using the connectivity im kleinen of $M$ it follows that $Q$ belongs to $C_{0}$ and that $C_{0}-Q$ is the sum of the two mutually separated sets $N \cdot C_{0}$ and $C_{0}[M-(N+Q)]$. Thus $Q$ is a cut point of $C_{0}$, contrary to the fact that $C_{0}$ is a nodular subset of $M$.

(3.3) If a subset $C$ of a connected and connected im kleinen set $M$ contains a non-cut point $P$ of $M$, then $C$ is a maximal nodular subset of $M$ if and only if $C$ is the set of all those points of $M$ which are not separated in $M$ from $P$ by any point of $M$.

The condition is sufficient. By (2.3), $C$ is closed in $M$. I shall now show 
that each component of $M-C$ has not more than one limit point in $C$. Suppose, on the contrary, that some component $N$ of $M-C$ has two limit points $A$ and $B$ in $C$. Since $M$ is connected im kleinen, it easily follows that there exist two connected and connected im kleinen subsets $I_{a}$ and $I_{b}$ of $M$ containing $A$ and $B$ respectively and such that $I_{a} \cdot N \neq 0 \neq I_{b} \cdot N$ and $I_{a} \cdot I_{b}=0$. There exist components $H_{x}$ and $H_{y}$ respectively of $I_{a} \cdot N$ and $I_{b} \cdot N$ having limit points $X$ and $Y$ respectively in $C$. Since $M$ is connected im kleinen, it follows that $N$ also is connected im kleinen; and if $H$ denotes the component of $N-\bar{H}_{x} \cdot N$ which contains $H_{y}$, then $\bar{H}_{x} \cdot N$ contains a point $Q$ which is a limit point of $H$. Now since $Q$ is not in $C$, there exists a point $Z$ of $M$ which separates $Q$ from $P$ in $M ; Z$ does not separate either $X$ or $Y$ from $P$ in $M$, because $X+Y \subset C$. Hence $Z$ must separate $Q$ from both $X$ and $Y$ in $M$. But clearly this is impossible, since $H_{x}+X+Q$ and $H+Y+Q$ are connected subsets of $M$ having only the point $Q$ in common. Thus the supposition that some component of $M-C$ has more than one limit point in $C$ leads to a contradiction.

Thus $C$ has the property mentioned in (2.1), and hence, by (2.2), $C$ is connected. Now $C$ can have no cut point. For suppose $C$ has a cut point $A$. Then if $A \neq P, A$ separates some point $Q$ of $C$ from $P$ in $C$; but since $A$ does not separate $Q$ from $P$ in $M$ (for $Q+P \subset C$ ), and $M$ is connected im kleinen, by (1.4) there exists a connected subset $H$ of $M-A$ containing both $Q$ and $P$. But by (2.1), $C \cdot H$ is connected; and since it contains both $P$ and $Q$, this contradicts the fact that $A$ separates $P$ and $Q$ in $C$. If $A=P$, then $P$ separates some two points $X$ and $Y$ of $C$ in $C$. But by hypothesis $P$ is not a cut point of $M$, and hence it does not separate $X$ and $Y$ in $M$. Hence, by (1.4), $M-P$ contains a connected subset $H$ containing both $X$ and $Y$. But by (2.1), $H \cdot C$ is connected; and since $H \cdot C \supset X+Y, P$ does not separate $X$ and $Y$ in $C$. Thus, in any case, the supposition that $C$ has a cut point leads to a contradiction. Therefore $C$ is a nodular subset of $M$. And since, as shown above, no component of $M-C$ has more than one limit point in $C$, it follows by (3.2) that $C$ is a maximal nodular subset of $M$.

The condition is also necessary. For let $C$ be any maximal nodular subset of $M$ containing a non-cut point $P$ of $M$. Let $C_{0}$ be the set of all those points of $M$ which are not separated in $M$ from $P$ by any point of $M$. Since $C$ is nodular, obviously $C \subset C_{0}$. Now it was just shown above that $C_{0}$ is a nodular subset of $M$. Therefore $C$ is not a proper subset of $C_{0}$, and hence $C=C_{0}$.

Note. In the first paragraph of the proof of (3.3) no use was made of the fact that $P$ is a non-cut point of $M$. Thus we have the following corollary.

(3.3a) If $P$ is any point of a connected and connected im kleinen set $M$ and $K$ 
is the set of all those points of $M$ not separated in $M$ from $P$ by any point of $M$, then $K$ has the property mentioned in (2.1). Hence $K$ is closed in $M$, connected, and connected im kleinen.

(3.4) $A$ nodular subset $C$ of a set $M$ is maximal if and only if $C$ is closed in $M$ and no component of $M-C$ has more than one limit point in $C$.

The condition is sufficient by (3.2). That it is necessary was proved incidentally in the proof of (3.3).

(3.5) Every maximal nodular subset $C$ of a set $M$ is closed in $M$, connected, and connected im kleinen; and $H \cdot C$ is either vacuous or connected, for every connected subset $H$ of $M$.

This follows at once from results (2.1), (2.2), (3.1), and (3.4).

(3.6) If $C_{0}$ contains more than one point and is any nodular subset of a set $M$, then there exists a maximal nodular subset $C$ of $M$ which contains $C_{0}$.

Since $C_{0}$ is connected and has no cut point, then* it can contain at most a countable number of cut points of $M$. Therefore it contains a non-cut point $P$ of $M$. And if $C$ denotes the set of all those points of $M$ which are not separated in $M$ by any point of $M$, then by (3.3), $C$ is a maximal nodular subset of $M$. Clearly $C_{0} \subset C$, because $C_{0}$ is nodular.

Definition. A point $P$ of a maximal nodular subset $C$ of a connected and connected im kleinen point set $M$ which is a non-cut point of $M$ will be called an internal point of $C$.

(3.7) No two maximal nodular subsets of a connected and connected im kleinen set $M$ can have more than one common point, nor can they have an internal point of either in common without being identical.

This follows at once from result (3.3).

(3.8) If $A$ and $B$ are any two points of a connected and connected im kleinen point set $M$ which are not separated in $M$ by any point of $M$, then there exists a maximal nodular subset of $M$ containing both $A$ and $B$.

Let $C$ be the set of all those points of $M$ which are not separated in $M$ from either $A$ or $B$ by any point of $M$. By (2.3), $C$ is closed in $M$. Clearly $C \supset A+B$. And by the same argument as given in the first paragraph of the proof of (3.3), with very slight modifications, it is shown that $C$ has the pro-

* See R. L. Moore, Concerning cut-points of continuous curves and of other closed and connected point-sets, Proceedings of the National Academy of Sciences, vol. 9 (1923), pp. 101-106; also see C. Zarankiewicz, loc. cit. 
perty mentioned in (2.1). Hence by (2.2), $C$ is connected. Now $C$ can have no cut point. For suppose $X$ is a cut point of $C$. Then $X$ separates some two points $U$ and $V$ of $C$ in $C$. Now $X$ cannot separate $U$ and $V$ in $M$; for if so, then $M-X=M_{u}(X)+M_{v}(X)$, and at least one of the sets $M_{u}(X)$ and $M_{v}(X)$ must contain a point of $A+B$; but then either $U$ or $V$ is separated in $M$ from one of the points $A$ and $B$ by the point $X$, contrary to the fact that $U+V \subset C$. Hence, by (1.4), $M-X$ contains a connected set $H$ containing $U+V$. But by (2.1), $C \cdot H$ is connected. Clearly this contradicts the supposition that $X$ separates $U$ and $V$ in $C$. Therefore $C$ has no cut point, and hence is a nodular subset of $M$. That $C$ is a maximal nodular subset of $M$ follows now from (3.4) and the fact that $C$ has the property of (2.1).

\section{Nodular Elements; Nodules}

Definition. The point $P$ of a connected and connected im kleinen point set $M$ will be called an end point of $M$ provided there exists an infinite sequence $X_{1}, X_{2}, X_{3}, \cdots$ of distinct points of $M$ converging to $P$ (i.e., having $P$ as its sequential limit point) and such that for each point $A$ of $M-P$, there exists an integer $k_{a}$ such that if $m>k_{a}$, then $X_{m}$ separates $A$ and $P$ in $M{ }^{*}$

(4.1) Every point of a connected and connected. im kleinen point set $M$ is either a cut point, an end point, or a point of some non-degenerate $\dagger$ nodular subset of $M$.

Let $P$ be any point of $M$ which is neither a cut point of $M$ nor a point of any non-degenerate nodular subset of $M$. I shall proceed to show that $P$ is an end point of $M$. Let $B$ be any point of $M-P$, and let $K$ be the set of all those points of $M$ which separate $P$ and $B$ in $M$. Now $K+B$ is not closed. For if so, then $K+B$ contains at least one limit point $Q$ of the component $N$ of $M-$ $(K+B)$ which contains $P$. Now $Q \neq B$; for if $Q=B$, then $K=0$ and, by (3.8), $P$ and $B$ lie together in a nodular subset of $M$, contrary to supposition. Hence $Q$ belongs to $K$, and therefore $M-Q=M_{p}(Q)+M_{b}(Q)$. But then $Q$ and $P$ are not separated in $M$ by any point $X$ of $M$; for if so, then since $X$ would

* For a point $P$ of any continuum $M$, the property mentioned in this definition is equivalent to the property of being a point of Menger order one of $M$, i.e., an end point of $M$ in the sense of Menger. This is not true for connected and connected im kleinen sets in general. However, this property is equivalent, in general, to the property of being a point of order 1 of $M$ in the sense that a monotone decreasing sequence $U_{1}, U_{2}, U_{3}, \cdots$ of neighborhoods of $P$ in $M$ exists such that the boundary of $U_{i}$ in $M$ is a single point $X_{i}$ and such that

$$
P=\prod_{1}^{\infty}\left(U_{i}+X_{i}\right) .
$$

† A set of points is degenerate or non-degenerate according as it does or does not reduce to a single point. This terminology is due to R. L. Moore. 
necessarily belong to $N$ and hence to $M_{p}(Q)$, and $M_{b}(Q)+Q$ is connected* and contains both $B$ and $Q$ but not $X$, it would follow that $X$ separates $B$ and $P$ in $M$ and hence belongs to $K$. This is absurd, since $X \subset N \subset M-K$. Thus no point of $M$ separates $Q$ and $P$ in $M$. Hence, by (3.8), $P$ and $Q$ lie together in a nodular subset of $M$, contrary to supposition. Therefore it follows that $K+B$ is not closed. Since, by (1.1), $(K+B)+P$ is closed, it follows that $P$ is a limit point of $K$. Accordingly there exists an infinite sequence $P_{1}, P_{2}, P_{3}, \cdots$ of points of $K$ converging to $P$. Now let $A$ be any point whatever of $M-P$. Clearly $\left(A+P+P_{1}+P_{2}+\cdots\right)-A=S$ is closed. Let $R$ be the component of $M-S$ containing $A$. Then since, by hypothesis, $P$ is not a cut point of $M$, manifestly there exists a point $P_{j}$ of $S$, distinct from $P$, which is a limit point of $R$. As $P_{j}$ belongs to $K$, then $M-P_{j}=M_{p}\left(P_{j}\right)+M_{b}\left(P_{j}\right)$. Since $\lim _{n \rightarrow \infty} P_{n}=$ $P$ and $\overline{M_{b}\left(P_{i}\right)} \cdot P=0$, there exists an integer $k_{a}$ such that for any integer $m>k_{a}, P_{m}$ belongs to $M_{p}\left(P_{j}\right)$. Let $m$ be any integer $>k_{a}$. Then since $R+$ $P_{i}+M_{b}\left(P_{j}\right)$ is connected and contains $A+B$ but not $P_{m}$, and $P_{m}$ separates $B$ and $P$ in $M$, it follows that $P_{m}$ separates $A$ and $P$ in $M$. Thus $P$ is an end point of $M$, and our theorem is proved.

Any non-degenerate maximal nodular subset of a set $M$ will be called a nodule of $M$. A subset $E$ of a set $M$ will be called a nodular element of $M$ provided that $E$ is either (a) a module of $M$, (b) a cut point of $M$, or (c) an end point of $M$. The nodular elements may also be defined as consisting of (1) the cut points of $M$ and (2) the maximal nodular subsets of $M$, the nondegenerate elements of (2) being the nodules of $M$ and the degenerate ones being the end points of $M . \dagger$

(4.2) If $N_{1}, N_{2}, N_{3}, \cdots$ is any sequence of nodules of a set $M$ having a sequential limiting set $L$, then $L \cdot M$ is either vacuous or it consists of a single point.

Suppose, on the contrary, that $L$ contains at least two points $A$ and $B$ of $M$. Then since $M$ is connected im kleinen, it readily follows that there exist two integers $i$ and $j$ and two mutually exclusive connected subsets $C_{a}$ and $C_{b}$ of $M$ such that $N_{i} \cdot C_{a} \neq 0 \neq N_{j} \cdot C_{a}$, and $N_{i} \cdot C_{b} \neq 0 \neq N_{j} \cdot C_{b}$. But then if $H$ denotes the component of $M-N_{i}$ which contains $N_{j}-N_{i} \cdot N_{j}\left(N_{i} \cdot N_{i}\right.$ contains at most one point), it is clear that $N_{i}$ contains at least two limit points of $H$, contrary to (3.4).

* Cf. Knaster and Kuratowski, Sur les ensembles connexes, Fundamenta Mathematicae, vol. 2 (1921), pp. 206-255.

† Compare with R. L. Moore, Concerning upper semi-continuous collections, Monatshefte für Mathematik und Physik, vol. 36 (1929), pp. 81-88, and also C. Kuratowski, Quelques applications d'éléments cycliques de M. Whyburn, Fundamenta Mathematicae, vol. 14 (1929), p. 138. 
(4.3) The nodules of any set $M$ are countable.

This follows at once from (4.2).

It should be noted that, under the conditions of $(4.2), \lim _{n \rightarrow \infty} \delta\left(N_{i}\right)$ may be $\neq 0$. The situation here differs from that existing in the case of the maximal cyclic curves of a continuous curve, due to the fact that the set $M$ may fail to be complete or locally compact in itself. It is also to be noted that, in (4.2), we may allow $N_{i}(i=1,2,3, \cdots)$ to be any connected collection of nodular elements of $M$ (see $\$ 6$ below), so long as $N_{i} \cdot N_{j}$ consists of at most one point, for each $i$ and $j$. This more general result follows by an argument very similar to the proof of (4.2), using (6.4) (below) instead of (3.4).

Proposition (4.1), together with (3.6), gives at once the fact that any connected and connected im kleinen point set may be decomposed into its nodular elements. Thus we may state the following theorem, which follows readily with the aid of (4.3).

(4.L) If $K, H$, and $N$, respectively, denote the sum of all the cut points, end points, and nodules of a set $M$, then (1) $K+H+N=M$, (2) $K \cdot N$ is countable, and (3) $K \cdot H=N \cdot H=0$.

\section{Simple Nodular chains}

Let $A_{0}$ and $B_{0}$ be any two nodular elements of a connected and connected im kleinen point set $M$, and let $A$ and $B$ respectively denote $A_{0}$ and $B_{0}$ or internal points of $A_{0}$ and $B_{0}$ according as $A_{0}$ and $B_{0}$ are single points or nodules of $M$. Then by the simple nodular chain of $M$ from $A_{0}$ to $B_{0}$ is meant the subset $X$ of $M$ defined in either of the two following equivalent ways.

(5.1) If $K$ is the set of all those points of $M$ separating $A$ and $B$ in $M$, then $X=K+A_{0}+B_{0}+$ all nodules of $M$ each of which contains exactly two points of $K+A+B$.

(5.2) $X$ is a subset of $M$ which is (a) connected, (b) the sum of the elements of some collection of nodular elements of $M$ including $A_{0}$ and $B_{0}$, and (c) irreducible with respect to properties (a) and (b).

We proceed to show the identity of the sets $X$ defined in (5.1) and (5.2). Denote these sets by $X_{1}$ and $X_{2}$ respectively. We first show that $X_{1}$ is a set satisfying conditions (a), (b), and (c) in (5.2). The set $X_{1}$ is connected; for if not, it is the sum of two mutually separated sets $U$ and $V$ each of which, it is easily seen, must contain a point of $K+A+B$. Since $K+A+B$ is closed, $U \cdot(K+A+B)=N_{1}$ and $V \cdot(K+A+B)=N_{2}$ are closed. One of these sets, say $N_{1}$, contains $A$. It follows by (1.2) that there exists a first point $Z$ of $N_{2}$ in the order from $A$ to $B$ in $M$ and likewise a last point $Y$ in $N_{1}$ which precedes 
$Z$ in the order $A, B$. But then $Y$ and $Z$ are not separated in $M$ by any point of $M$, since there is no point of $K$ between them. Accordingly, by (3.8), $M$ contains a nodule $N$ containing $Y+Z$. Clearly $N$ contains no other point of $K+A+B$. But then $N \subset X_{1}$, and this is impossible, since $N$ is connected and $U$ and $V$ are mutually separated. Thus $X_{1}$ is connected and hence satisfies (a). It satisfies (b) by definition, since $X_{1}=K+A_{0}+B_{0}+$ nodules of $M$. Suppose, contrary to (c), that $X_{1}$ contains a proper subset $Y$ having properties (a) and (b). Clearly $Y \supset K$; but since $Y$ is not $=X_{1}$, there exists a point $Z$ of $X_{1}-Y$ which must belong to a nodule $N$ of $M$ which contains two points $U$ and $V$ of $K+A+B$. Now $Y \cdot N \supset U+V$ and since, by (3.5), $Y \cdot N$ is connected, it must contain at least one internal point of $N$. But then, by (3.7), $N \subset Y$, contrary to supposition. Thus $X_{1}$ has property (c). Hence $X_{1}$ is a set having properties (a), (b), and (c) in (5.2). That $X_{1}$ is identical with every set having these properties follows by almost the same argument as just given to show that it has property (c). Thus $X_{1}=X_{2}$.

The following two propositions follow readily from the results just established above.

(5.3) For every two nodular elements $N_{1}$ and $N_{2}$ of a set $M$, there exists in $M$ one and only one simple nodular chain $X$ from $N_{1}$ to $N_{2}$.

(5.4) Every simple nodular chain of a set $M$ is closed in $M$, connected, and connected im kleinen.

Note. For the case where $M$ is closed, i.e., where $M$ is a continuous curve, the nodular elements of $M$ coincide with the cyclic elements of $M$, and the simple nodular chain in $M$ between two nodular elements $N_{1}$ and $N_{2}$ of $M$ is identical with the simple cyclic chain in $M$ from $N_{1}$ to $N_{2}$ (see my paper Concerning the structure of a continuous curve, loc. cit.) and with the arccurve $A B$, where $A$ and $B$ are points of $N_{1}$ and $N_{2}$ respectively and are non-cut points of $M$ in case $N_{1}$ or $N_{2}$ is non-degenerate, in the sense of W. L. Ayres.* Additional properties of nodular chains similar to those of simple cyclic chains, or arc-curves, could be stated and proved in an analogous way; but to save space the reader is simply referred to $\$ 2$ of the above mentioned paper of the author's and to the slightly later paper of Ayres.

\section{CONNECTED COLLECTIONS OF NODULAR ELEMENTS}

A connected subset of a connected and connected im kleinen point set $M$ which is the sum of the elements of some collection of nodular elements of $M$ will be called a connected collection of nodular elements of $M$.

* Cf. Concerning arc-curves and basic sets of a continuous curve, these Transactions, vol. 30 (1928), pp. 567-578. 
(6.1) If $N_{1}$ and $N_{2}$ are nodular elements of a set $M$ belonging to a connected collection $H$ of nodular elements of $M$, then $H$ contains the simple nodular chain $X$ of $M$ between $N_{1}$ and $N_{2}$.

Let $A$ and $B$ be points of $N_{1}$ and $N_{2}$ respectively, and let them be internal points if $N_{1}$ and $N_{2}$ or either is a nodule of $M$. Let $K$ be the set of all points of $M$ each of which separates $A$ and $B$ in $M$. Now suppose that $H$ does not contain $X$. Then $X-H \cdot X$ contains a point $P$. Since $H$ is connected and contains $A+B$, therefore it contains $K$. Hence, by (5.1), $P$ belongs to a nodule $N$ of $M$ containing two points $U$ and $V$ of $K+A+B$. But since, by (3.5), $N \cdot H$ is connected, then $H$ contains an internal point of $N$; and hence, by (3.7), $H \supset N \supset P$, contrary to supposition. This contradiction proves our theorem.

(6.2) If $H$ is any connected collection of nodular elements of a set $M$, then every point of $\bar{H} \cdot M-H$ is a nodular element of $M$ (and hence is either a cut point or an end point of $M$ ).

We proceed to show that each point of $\bar{H} \cdot M-H$ is either a cut point or an end point of $M$. If this is not so, then some point $P$ of $\bar{H} \cdot M-H$ is an internal point of some nodule $N$ of $M$. Now $H \cdot N \neq 0$; for if not, it would follow by (3.4) that one and only one point $Q$ of $N$, different from $P$, is a limit point of $H$. Hence $H$ contains a point $Q$ of $N$; and since $P$ is a limit point of $H$, it follows readily that $N \cdot H$ contains at least two points. And since, by (3.5), this set $N \cdot H$ is connected, therefore it contains an internal point of $N$. But then, by (3.7), $H \supset N \supset P$, contrary to the fact that $P \subset$ $\bar{H} \cdot M-H$. Thus $P$ is either a cut point or an end point of $M$, and our theorem is established.

(6.3) Under the hypothesis of (6.2), $\bar{H} \cdot M$ is a connected collection of nodular elements of $M$.

(6.4) If $H$ is any connected collection of nodular elements of a set $M$, and $N$ is any component of $M-H$, then $M \cdot \bar{H} \cdot \bar{N}$ consists of exactly one point.

That this set contains at least one point follows from the fact that $M$ is connected im kleinen. Suppose, contrary to (6.4), that it contains two pcints $A$ and $B$. Since $H+A+B$ and $N+A+B$ are connected subsets of $M$, ard $N \cdot H=0$, clearly no point of $M$ separates $A$ and $B$ in $M$. Consequently, by (3.8), $A+B$ lies in a single nodule $C$ of $M$. By (3.5), $C \cdot(H+A+B)$ is connected, and since it contains $A+B$, it follows by (3.7) that $H \supset C$. But then $(N+A+B) \cdot C=A+B$, a disconnected set, contrary to (3.5). Thus our theorem is established. 
(6.5) If $H$ is any connected collection of nodular elements of a set $M$ and $W$ is any connected subset of $M$, then $H \cdot W$ is either vacuous or connected.

Let $A$ and $B$ be any two points of $H \cdot W$, and let $A_{0}$ and $B_{0}$ respectively denote nodular elements of $M$ belonging to $H$ and containing $A$ and $B$. By (6.1), the simple nodular chain $X$ in $M$ from $A_{0}$ to $B_{0}$ is a subset of $H$. Now since $X$ is closed in $M$ and is a conrected collection of elements of $M$, it follows by (6.4) and (2.1) that $W \cdot X$ is connected. And since $W \cdot X$ contains $A+B$ and is a subset of $H \cdot W$, and $A$ and $B$ are any two points of $H \cdot W$, it follows that $H \cdot W$ is connected.

(6.6) Every connected collection $H$ of nodular elements of a set $M$ is connected im kleinen.

Since $M$ is connected im kleinen, then for each point $P$ of $M$ and each $\epsilon>0$, a $\delta_{\epsilon}>0$ exists such that for each point $Q$ of $H$ with $\rho(Q, P)<\delta_{\epsilon}$, a connected subset $C$ of $M$ exists with $P+Q \subset C$ and $\delta(C)<\epsilon$. And since, for every such set $C$, by (6.5), $C \cdot H$ is connected, it follows that $H$ is connected im kleinen.

\section{SOME A.PPLICATIONS; DENODULAR SETS}

In this section the results of the preceding sections will be applied to yield some interesting (new) results concerning the structure of certain special types of connected and connected im kleinen sets.

A set $M$ which satisfies any one of the following equivalent conditions will be called a denodular set.

(7.1) $M$ contains no nodules, i.e., all its nodular elements are points.

(7.2) Every two points of $M$ are separated in $M$ by some third point of $M$.

(7.3) Every point of $M$ is either a cut point or an end point.

The equivalence of these three properties is easily established with the aid of the results of the preceding sections.

(7.4) Every connected set $H$ of degenerate nodular elements of a set $M$ (i.e., every connected set of cut points and end points of $M)$ is a denodular set.

By (6.6), $H$ is connected im kleinen and hence is itself a set $M$. That $H$ is denodular follows immediately now from (7.1).

(7.5) Every denodular set $M$ is arcwise connected; indeed it is uniquely arcwise connected.

Let $A$ and $B$ be any two points of $M$. By (5.2), $M$ contains a simple 
nodular chain $X$ from $A$ to $B$. By (5.1) it follows that $X \equiv K+A+B$, where $K$ is the set of all points of $M$ separating $A$ and $B$ in $M$. And since by (1.1), $K+A+B$, or $X$, is compact and closed, it is then a simple continuous arc from $A$ to $B$. That $X$ is the only arc in $M$ from $A$ to $B$ follows from (5.3).

(7.6) Every connected set of cut points and end points of any connected and connected im kleinen set $M$ is uniquely arcwise connected.

This is an immediate consequence of (7.4) and (7.5).

(7.7) Lemma. Let $A$ and $B$ be any two points of a connected and arcwise connected im kleinen set $M$, let $C$ be the collection of all connected subsets of $M$ containing both $A$ and $B$, let $T$ be the collection of all arcs in $M$ from $A$ to $B$, and let $L$ be the greatest lower bound of the number aggregate $[\delta(c)]$, where $c$ is an element of $C$. Then $L$ is also the greatest lower bound for the aggregate $[\delta(t)]$, where $t$ is an element of $T$.

Let $\epsilon$ be any positive number. By the hypothesis, a connected subset $c$ of $M$ exists with $\delta(c)<L+\epsilon / 4$. Since $M$ is connected im kleinen, then for each point $X$ of $c$, a connected open subset $H_{x}$ of $M$ exists containing $x$ and with $\delta\left(H_{x}\right)<\epsilon / 4$. Then if $H=\sum H_{x}$, it is clear that $H$ is a connected open subset of $M$, and $\delta(H)<L+\epsilon$. But* $H$ is arcwise connected; and since it contains $A+B$, it therefore contains an $\operatorname{arc} t$ in $M$ from $A$ to $B$. Then $t$ is an element of $T$ and $\delta(t)<L+\epsilon$; accordingly, $L$ is the greatest lower bound for $[\delta(t)]$, and our lemma is proved.

(7.8) If every nodule of a set $M$ is arcwise connected im kleinen, then $M$ itself is arcwise connected im kleinen. Hence $M$ is also arcwise connected.

Let $A$ be any point of $M$. For each $\epsilon>0$, a $\delta_{\epsilon a}>0$ exists such that for each point $x$ of $M$ with $\rho(A, x)<\delta_{\epsilon a}$ a connected subset $C$ of $M$ exists with $A+x \subset C$ and $\delta(C)<\epsilon / 8$. Let $B$ be any such point $x$ of $M$. Let $K$ be the set of all points of $M$ separating $A$ and $B$ in $M$, and let $X$ be the simple nodular chain in $M$ from $A$ to $B$. Let the nodules of $M$ in $X$ be ordered (they are countable by (4.3)): $N_{1}, N_{2}, N_{3}, \cdots$. For each $i>0$, let $A_{i}$ and $B_{i}$ be the two points (see (5.1)) of $N_{i} \cdot(K+A+B)$, and let $L_{i}$ be the greatest lower bound of $\left[\delta\left(c_{i}\right)\right]$ where $C_{i}$ is the collection of all connected subsets of $N_{i}$ containing $A_{i}+B_{i}$, and $c_{i}$ is an element of $C_{i}$. For each $i$, by (7.7), $N_{i}$ contains an arc $t_{i}$ from $A_{i}$ to $B_{i}$ with

* See G. T. Whyburn, Concerning the complementary domains of continua, Annals of Mathematics, vol. 29, pp. 399-411, Theorem 12. This theorem, together with its proof, holds equally well in the space here considered. 


$$
\delta\left(t_{i}\right)<L_{i}+\epsilon /(8 i) .
$$

Let

$$
W=K+A+B+\sum_{i=1}^{\infty} t_{i}
$$

Then $W$ is an arc from $A$ to $B$, as will now be demonstrated. Clearly $W$ is connected and irreducibly connected between $A$ and $B$. It is also closed; for if not, then since $K+A+B$ is closed, there would exist a positive number $d$ and an infinite sequence $t_{n_{1}}, t_{n_{2}}, \cdots$ of the arcs $\left[t_{i}\right]$ with

$$
\delta\left(t_{n_{\mathrm{i}}}\right)>d,
$$

for every $i$, and furthermore such that there exists a sequential limiting set $L$ of this sequence containing a point $P$ of $K+A+B$. Since $M$ is connected im kleinen at $P$, a $\delta>0$ exists such that for each point $x$ of $M$ with $\rho(P, x)<\delta$, a connected subset $H$ of $M$ exists containing $P+x$ and with $\delta(H)<d / 8$. Since, as is easily established, $\lim _{i \rightarrow \infty} \rho\left(A_{i}, B_{i}\right)=0$, and since by $(4.2), L \cdot M=$ $P$, it readily follows that an integer $n_{j}$ exists such that

$$
\epsilon /\left(8 n_{j}\right)<d / 8
$$

and such that $\rho\left(A_{n_{j}}+B_{n_{j}}, P\right)<\delta$. There exists, then, a connected subset $B$ of $M$ with $\left(A_{n_{j}}+B_{n_{j}}+P\right) \subset H$ and $\delta(H)<d / 4$. Now by $(3.5), H \cdot N_{n_{j}}$ is connected, and since $\delta\left(H \cdot N_{j}\right)<d / 4$ and $A_{n_{j}}+B_{n_{j}} \subset H \cdot N_{j}$, it follows that $L_{n_{j}} \leqq d / 4$. Hence by (i) and (iv), it follows that

$$
\delta\left(t_{n_{j}}\right)<L_{n_{j}}+\epsilon /\left(8 n_{j}\right) \leqq d / 4+d / 8<d .
$$

But (iii) and (v) are contradictory. Therefore $W$ is closed and hence is an arc from $A$ to $B$. It remains to show that $\delta(W)<\epsilon$. Now since a connected subset $C$ of $M$ exists with $A+B \subset C$ and $\delta(C)<\epsilon / 8$, and since by (3.5), $C \cdot N_{i}$ is connected and $C \cdot N_{i} \supset A_{i}+B_{i}$ for every $i$, it follows that $L_{i}<\epsilon / 8$ for every $i$. Hence by (i), $\delta\left(t_{i}\right)<\epsilon / 8+\epsilon /(8 i) \leqq \epsilon / 4$ for every $i$. Now $\delta(K+A+B)$ $<\epsilon / 8$, for $K+A+B \subset C$; and since every arc $t_{i}$ has two points in $K+A+B$, it follows from (ii) that

$$
\delta(W)<2 \cdot \epsilon / 4 \epsilon / 8<\epsilon .
$$

Thus $M$ is arcwise connected im kleinen at every point. That $M$ is also arcwise connected follows now by the theorem of the author's quoted above.

Definition. A connected and connected im kleinen point set $M$ will be said to be locally denodular if for each point $P$ of $M$ a neighborhood $R$ of $P$ exists such that the component of $M \cdot R$ containing $P$ is a denodular set. This 
notion is analogous to Menger's notion of a Baum im kleinen.*

(7.9) If every nodule of a set $M$ is locally denodular, then $M$ is arcwise connected and arcwise connected im kleinen.

It follows by (7.5) that every nodule of $M$ is arcwise connected im kleinen. Hence by (7.8), $M$ is arcwise connected im kleinen and arcwise connected.

Definition. A collection $G$ of subsets of a set $M$ will be called an $E$ collection provided that if $g_{1}, g_{2}, g_{3}, \ldots$ is any sequence of elements of $G$ having a non-vacuous sequential limiting set $L$ with $L \cdot M \neq 0$, then $\lim _{n \rightarrow \infty}$ $\delta\left(g_{n}\right)=0$.

As pointed out above, the collection $G$ of all the nodules of a set $M$ may fail to be an $E$-collection, although it is true that the collection of all maximal cyclic curves of a continuous curve is an $E$-collection. As might be expected, it is true that if we stipulate that the collection $G$ of nodules is an $E$-collection, we get a set $M$ which is more completely analogous, from the standpoint of its nodular elements, to a continuous curve; and some known theorems about continuous curves extend to such sets $M$ which would not extend to sets $M$ in general. As an example, we state the following one.

(7.10) If the collection of all nodules of a set $M$ is an E-collection, then in order that $M$ should be arcwise connected it is necessary and sufficient that each nodule of $M$ be arcwise connected.

This theorem is proved by a rather obvious modification of the proof of (7.8). A number of other theorems of the same type could also be stated for such sets $M$. The reader is referred to $\S 6$ of my paper Concerning the structure of a continuous curve. It is to be noted that if $M$ is any connected subset of a Menger regular curve, then it is connected im kleinen and the collection of all nodules of $M$ has property $E$.

\section{LiNEAR ARRAYS OF NODULAR ELEMENTS}

Definition. The connected collection $H$ of nodular elements of a set $M$ will be called a linear array of elements of $M$ provided every nodular element of the set $H$ itself which cuts $H$ cuts it into exactly two components. It is readily seen that not more than two nodular elements of a linear array $H$ of elements can fail to cut $H$. If every element of the linear array $H$ cuts $H$, then $H$ is called an open chain of nodular elements of $M$; if $H$ itself has more than one nodular element, and if every element, save one element $E$, of $H$ cuts $H$, then $H$ will be called a radial chain of nodular elements of $M$ and will

\footnotetext{
* Cf. Über reguläre Baumkurven, Mathematische Annalen, vol. 96 (1926), pp. 572-582.
} 
be said to emerge from $E$; if every element, save two elements $A_{0}$ and $B_{0}$, of $H$ cuts $H$, then $H$ is identically the simple nodular chain in $M$ from $A_{0}$ to $B_{0}$. (See $\$ 5$.)

If $E$ is any nodular element of an open chain $L$ of nodular elements of a set $M$, then $L$ is the sum of two radial chains $r_{1}$ and $r_{2}$ emerging from $E$ and having only $E$ in common; furthermore, there exist two sequences $A_{1}, A_{2}$, $A_{3}, \cdots$ and $B_{1}, B_{2}, B_{3}, \cdots$ of cut points of $L$ belonging to $r_{1}$ and $r_{2}$ respectively and such that neither $\left[A_{i}\right]$ nor $\left[B_{i}\right]$ has a limit point in $L$ and if, for each $i, X_{i}$ is the simple nodular chain in $M$ from $A_{i}$ to $B_{i}$, then $L=\sum X_{i}$ and, for each $i, X_{i} \subset X_{i+1}$. If $L$ is any open chain in a set $M$, then $M \cdot \bar{L}-L$ contains at most two points; and $M \cdot \bar{L}$ is either a simple nodular chain, a radial chain, or an open chain in $M$ according as $M \cdot \bar{L}-L$ contains two points, one point, or no points. If $L$ is any open chain of nodular elements of a set $M$, then there exists in $M$ a maximal open chain of nodular elements containing $L$, i.e., an open chain of elements which is saturated with respect to the property of being an open chain of nodular elements of $M$; if $r$ is any radial chain of nodular elements of $M$, then there exists in $M$ either a maximal radial chain or a maximal open chain (sometimes both) containing $r$; if $X$ is any simple nodular chain in $M$, then there exists in $M$ either a maximal simple nodular chain, a maximal radial chain or a maximal open chain containing $X$. If $U$ is any maximal simple nodular chain of a set $M$, or is any maximal radial chain or maximal open chain of $M$ which is closed in $M$, then $U$ is a maximal linear array in $M$, i.e., no linear array in $M$ contains $U$ as a proper subset. Clearly any maximal linear array in $M$ must be closed in $M$, and indeed, if $H$ is any linear array of nodular elements in a set $M$, then there exists in $M$ a maximal linear array which contains $H$. The facts just enumerated can be established quite readily by methods of argument which are well known in point set theory.

If $M$ is a denodular set, it is easily seen that every open chain of nodular elements of $M$ is an ordinary open curve, or what is equivalent, an arc segment, every radial chain in $M$ is an ordinary ray, and every simple nodular chain is an ordinary simple continuous arc. Thus if $M$ is denodular, and $L$ is any open curve in $M$, then $M \cdot \bar{L}-L$ is either vacuous, one point, or two points. And if $L$ is any maximal open curve in $M$, then each point, if any, of $M \cdot \bar{L}-L$ is an end point of $M$; and $\bar{L} \cdot M$ is either $\equiv L$, or is a maximal ray in $M$, or is a maximal arc in $M$, according as $M \cdot \bar{L}-L$ is vacuous, one point, or two points. Furthermore, if $L$ is any open curve in $M$, there exists in $M$ a maximal open curve containing $L$; if $r$ is any ray in $M$, there exists in $M$ either a maximal ray or a maximal open curve containing $r$; and if $A B$ is any arc in $M$, there exists in $M$ either a maximal arc, a maximal ray, or a maximal open curve containing $A B$. 
(8.1) Let $M$ be any connected and connected im kleinen set. Then there exists in $M$ a countable sequence of point sets $S_{1}, S_{2}, S_{3}, \ldots$ such that (1) $S_{1}$ is a maximal linear array of nodular elements of $M$, (2) for each $n>1, S_{n}$ has exactly one point $A_{n}$ in common with $\sum_{i=1}^{n-1} S_{i}, S_{n}-A_{n}$ is a subset of some componeni $N_{n}$ of $M-\sum_{i=1}^{n-1} S_{i}$ which has $A_{n}$ as its limit point in $\sum_{i=1}^{n-1} S_{i}$, and $S_{n}$ is a maximal linear array of nodular elements in the set $N_{n}+A_{n}$. [hence either $S_{n}$ is a radial chain emerging from $A_{n}$ (and lying otherwise in $N_{n}$ ) or else there exists a nodular element $B_{n}$ such that $S_{n}$ is the simple nodular chain in $M$ from $A_{n}$ to $B_{n}$ (a single nodule may be a simple nodular chain)], and (3) every point, if any, of $M-\sum_{1}^{\infty} S_{n}$ is an end point of $M$.

Using the fact that $M$ is separable, this proposition may be established on the basis of the preceding results by an argument which is by no means difficult.

(8.2) Let $G$ be the sum of all cut points and all the end points of any connected and connected im kleinen point set $M$. Then there exists a subset $H$ of the universal acyclic continuous curve* $W$ of Wazewski and a biunivalued transformation $T$ such that (1) $T(G)=H$, (2) $T$ is continuous, and $T^{-1}$ is continuous over every arc in $H$.

This proposition may be proved on the basis of the properties of the nodular elements of $M$ by an argument not essentially different from that used in my paper On the set of all cut points of a continuous curve $\dagger$ to prove an analogous though stronger theorem concerning the set of all cut points and end points of a continuous curve. It should be noted that it is not always possible for the transformation $T$ to be bicontinuous, even in case $M$ is a denodular set. For example, let $A$ denote the $X$-axis, and for each integer $n \neq 0$, let $L_{n}$ denote the line $x=1 / n$; and finally, let $M=A+\sum L_{n}$. Then clearly the set $G$, which is $\equiv M$, is not. homeomorphic with any subset of an acyclic continuous curve. It seems to be true, however, that for sets $M$ having the property that if $X_{1}, X_{2}, \cdots$ is any sequence of mutually exclusive simple nodular chains in $M$ having a sequential limiting set which contains a point of $M$, then $\lim _{n \rightarrow \infty} \delta\left(X_{n}\right)=0$, it is true that the set $G$ is homeomorphic with some subset $H$ of an acyclic continuous curve.

If $M$ is denodular, then $G \equiv M$ and proposition (8.2) gives the following interesting corollary:

* This is a compact plane acyclic continuous curve $W$ such that every acyclic continuous curve is homeomorphic with some subset of $W$. See T. Wazewski, Sur les courbes de Jordan non renfermant aucune courbe simple fermée de Jordan, Annales de la Société Polonaise Mathématique, vol. 2 (1923), p. 169.

† Fundamenta Mathematicae, vol. 15, pp. 185-194. 
(8.2a) For every denodular set $D$ there exists a subset $H$ of an acyclic continuous curve and a biunivalued transformation $T$ such that $(1) T(D)=H$, and (2) $T$ is continuous, and $T^{-1}$ is continuous over every arc in $H$.

\section{CoNClusion; SOME UNSOLVED PROBLEMS}

The author is quite well aware that the above group of propositions is by no means exhaustive on this subject. An attempt has been made, in general, only to include those theorems and proofs which are essential in the construction of the foundation for the central idea of the paper. One who has followed through the results in the above sections and who is familiar with the cyclic elements of a continuous curve cannot fail, I believe, to see the pattern, beautiful in and because of its simplicity, of the connected and connected im kleinen point set neatly arranged into nodular elements with respect to which the structure of the whole set is so like that of an acyclic continuous curve. Once this pattern is grasped, it is a simple matter to observe and establish many other properties of and relations among the nodular elements. In the course of this investigation, however, there have occurred to me two problems of an entirely different nature, which I have been unable to solve and whose solution would, I believe, be of considerable interest and importance. They are as follows.

(9.1) Problem. Do the nodules of a set $M$ possess any property which might be called "cyclic"? It is known, of course, that if $M$ is a continuous curve, then every nodule $C$ of $M$, or what is the same thing, every maximal cyclic curve $C$ of $M$ is cyclicly connected, i.e., every two points of $C$ lie together on a simple closed curve in $C$. Do we have any sort of analogue to this property in a set $M$ in general? Or, more specifically, if a set $M$ has no cut point, then is it true that for every two points $A$ and $B$ of $M$, two connected subsets $B$ and $K$ of $M$ exist with $H \cdot K \equiv A+B$ ? If so, can $H$ and $K$ be chosen so that $H-(A+B)$ and $K-(A+B)$ are mutually separated? The terms nodule and nodular element were introduced and used in this paper because of the lack of knowledge of the existence of any such property to justify the use of the terms cyclic subset and cyclic element, as was done for a continuous curve.

(9.2) Problem. In order that the bounded set $M$ lying in a plane $Z$ should cut $Z$ in the weak sense, is it necessary (it is sufficient) that some nodule of $M$ cut $Z$ in the weak sense? Or less generally, is it true that no bounded denodular set $M$ lying in a plane $Z$ can cut $Z$ in the weak sense? It also seems probable to me that if the set $M$ lying in a plane $Z$ does not cut $Z$ in the weak sense, then either $M$ is denodular or else, for each nodule $N$ of $M$, there exists a domain $R$ such that $R \subset N \subset \bar{R}$.

UNIVERSITY OF TEXas, Austin, Texas 